
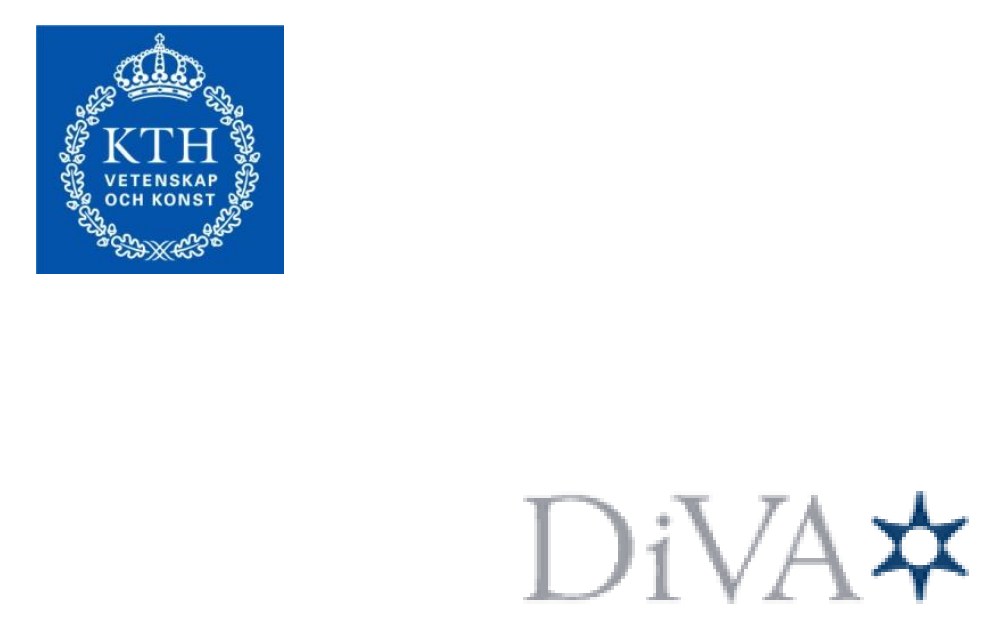

http://kth.diva-portal.org

This is an author produced version of a paper published in 10th World Congress on Intelligent Control and Automation (WCICA), 2012.

This paper has been peer-reviewed but does not include the final publisher proofcorrections or proceedings pagination.

(C) 2012 IEEE. Personal use of this material is permitted. Permission from IEEE must be obtained for all other uses, in any current or future media, including reprinting/republishing this material for advertising or promotional purposes, creating new collective works, for resale or redistribution to servers or lists, or reuse of any copyrighted component of this work in other works.

Citation for the published paper:

Youcheng Lou, Guodong Shi, Karl Henrik Johansson and Yiguang Hong. Reaching optimal consensus for multi-agent systems based on approximate projection.

10th World Congress on Intelligent Control and Automation (WCICA), 2012.

Access to the published version may require subscription.

Published with permission from: IEEE 


\title{
Reaching Optimal Consensus for Multi-agent Systems Based on Approximate Projection*
}

\author{
Youcheng Lou ${ }^{1}$, Guodong Shi ${ }^{2}$, Karl Henrik Johansson ${ }^{2}$ and Yiguang Hong ${ }^{1}$ \\ ${ }^{1}$ Key Lab of Systems and Control \\ Institute of Systems Science \\ Chinese Academy of Sciences \\ Beijing, 100190, China \\ louyoucheng@amss.ac.cn,yghong@iss.ac.cn \\ ${ }^{2}$ ACCESS Linnaeus Center \\ School of Electrical Engineering \\ Royal Institute of Technology \\ Stockholm, 10044, Sweden \\ guodongs@kth.se,kallej@kth.se
}

\begin{abstract}
In this paper, we propose an approximately projected consensus algorithm for a multi-agent system to cooperatively compute the intersection of several convex sets, each of which is known only to a particular node. Instead of assuming the exact convex projection, we allow each node to just compute an approximate projection. The communication graph is directed and time-varying, and nodes can only exchange information via averaging among local view. We present sufficient and/or necessary conditions for the considered algorithm on how much projection accuracy is required to ensure a global consensus within the intersection set, under the assumption that the communication graph is uniformly jointly strongly connected. A numerical example indicates that the approximately projected consensus algorithm achieves better performance than the exact projected consensus algorithm. The results add the understanding of the fundamentals of distributed convex intersection computation.
\end{abstract}

Index Terms-Multi-agent systems, approximate projection, intersection computation, optimal consensus

\section{INTRODUCTION}

In recent years, dynamics on large-scale networks has drawn various research attention in different areas including engineering, computer science, and social science. Cooperative control of a group of autonomous agents fully employs local information exchange and distributed protocol design to accomplish collective tasks such as agreement, formation, and aggregation [7], [8], [18], [15], [16], [33], [19], [17], [11], [12]. Moreover, in parallel computation, load-balance problems require realtime balance of the load from different computing resources [9], [10]. Additionally, a central problem of opinion dynamics in social networks is how the agreement is achieved via individual belief exchange processes [13], [14]. A fundamental question in these problems is, how consensus can be guaranteed based on local information exchange, timevarying node interconnections and limited knowledge of the global objective.

\footnotetext{
${ }^{*}$ This work is partially supported by NSF Grant 61174071 , the Knut and Alice Wallenberg Foundation and the Swedish Research Council.
}

Various distributed optimization problems arise for consensus with particular optimization purpose in practice. Minimizing a sum of convex functions, where each component is known only to a particular node, has attracted much attention recently, due to its simple formulation and wide applications [22], [20], [21], [26], [27], [23], [31], [30], [28], [29], [32], [25], [24]. The key idea is that properly designed distributed control protocols or computation algorithms can lead to a collective optimization, based on simple exchanged information and individual optimum observation. Subgradient-based incremental methods were established via deterministic or randomized iteration, where each node is assumed to be able to compute a local subgradient value of its objective function [20], [21], [26], [22], [25], [24]. Non-subgradientbased methods also showed up in the literature. For instance, a non-gradient-based algorithm was proposed, where each node starts at its own optimal solution and updates using a pairwise equalizing protocol [28], [29], and later an augmented Lagrangian method was introduced in[32].

In particular, if the optimal solution set of its own objective can be obtained for each node, the considered optimization problem is then converted to a set intersection computation problem when we additionally assume there is a nonempty intersection among all solution sets [31], [30], [27]. In fact, convex intersection computation problem is a classical problem in the optimization study [34], [35], [36]. The so-called "alternating projection algorithm" was a standard centralized solution, where projection is carried out alternatively onto each set [34], [35], [36]. Then the "projected consensus algorithm" was presented as a decentralized version of alternating projection algorithm, where each node alternatively projects onto its own set and averages with its neighbors, and comprehensive convergence analysis was given for this projected algorithm under time-varying directed interconnections in [27]. Following this work, a flipcoin algorithm was introduced when each node randomly chooses projection or averaging by Bernoulli processes, and 
almost sure convergence was shown for the system to reach an optimal consensus in [31]. A dynamical system solution was given in [30], where the network reaches a global optimal consensus by a simple continuous-time control. In all these algorithms, each node needs to know the exact convex projection of its current state onto its objective set [31], [30], [27].

However, in practice, the exact convex projection is usually hard to compute due to the common environmental noise and computation inaccuracy. In this paper, we therefore propose an approximately projected consensus algorithm (APCA) to solve the convex intersection computation problem. Instead of assuming the exact convex projection, we allow each node to just compute an approximate projection point which locates in the intersection of the convex cone generated by the current state and all directions with the exact projection direction less than some angle and the half-space containing the current state with its boundary being a supporting hyperplane to its own set at its exact projection point onto its set. The communication graph is supposed to be directed and time-varying. With uniformly jointly strongly connected conditions, we show that the whole network can achieve a global consensus within the intersection of all convex sets when sufficient projection accuracy can be guaranteed. For a special approximate projection case when the nodes can get the exact direction of the projection, a necessary and sufficient condition is given on how much projection accuracy is critical to ensure a global intersection computation. A numerical example is also given, and surprisingly, the APCA sometimes achieves better performance for convergence than the exact projected consensus algorithm.

The paper is organized as follows. Section II gives some basic concepts on graph theory and convex analysis. Section III introduces the network model and formulates the problem of interest. Section IV presents the main results and convergence analysis for the APCA. Section V gives a numerical example and finally, Section VI shows some concluding remarks.

\section{Preliminaries}

In this section, we introduce preliminary knowledge on graph theory [5] and convex analysis [1].

\section{A. Graph Theory}

A directed graph (digraph) $\mathcal{G}=(\mathcal{V}, \mathcal{E}, A)$ consists of node set $\mathcal{V}=\{1,2, \ldots, n\}$, arc set $\mathcal{E} \subseteq \mathcal{V} \times \mathcal{V}$ and an adjacency matrix $A=\left[a_{i j}\right]_{n \times n}$ with nonnegative adjacency elements $a_{i j}$. The element $a_{i j}$ of matrix $A$ associated with arc $(i, j)$ is positive if and only if $(i, j) \in \mathcal{E} . \mathcal{N}_{i}$ denotes the set of neighbors of node $i$, that is, $\mathcal{N}_{i}=\{j \in \mathcal{V} \mid(i, j) \in \mathcal{E}\}$. In this paper, we assume $(i, i) \in \mathcal{E}$ for all $i$. A path from $i$ to $j$ in digraph $\mathcal{G}$ is a sequence $\left(i_{0}, i_{1}\right),\left(i_{1}, i_{2}\right), \ldots,\left(i_{p-1}, i_{p}\right)$ of arcs with $i_{0}=i$ and $i_{p}=j . \mathcal{G}$ is said to be strongly connected if there exists a path from $i$ to $j$ for each pair of nodes $i, j \in \mathcal{V}$.

\section{B. Convex Analysis}

A function $f(\cdot): R^{m} \rightarrow R$ is said to be convex if $f(\lambda x+$ $(1-\lambda) y) \leq \lambda f(x)+(1-\lambda) f(y)$ for all $x, y \in R^{m}$ and $0<\lambda<1$. A function $f$ is said to be concave if $-f$ is convex.

A set $K \subset R^{m}$ is said to be convex if $\lambda x+(1-\lambda) y \in K$ for any $x, y \in K$ and $0<\lambda<1$ and is said to be a convex cone if $\lambda_{1} x+\lambda_{2} y \in K$ for any $x, y \in K$ and $\lambda_{1}, \lambda_{2} \geq 0$. For a set $K, c o(K)$ denotes the convex set consisting of all finite convex combinations of elements in $K$. For a closed convex set $K$ in $R^{m}$, we can associate to any $x \in R^{m}$ a unique element $P_{K}(x) \in K$ satisfying $\left|x-P_{K}(x)\right|=\inf _{y \in K}|x-y|$, which is denoted as $|x|_{K}$, where $|\cdot|$ denotes the Euclidean norm and $P_{K}$ is the projection operator onto $K$.

For a closed convex set $K$, if $x \notin K$, then by the supporting hyperplane theorem, there is a supporting hyperplane to $K$ at $P_{K}(x)$. The angle between vectors $a$ and $b$ is denoted as $\operatorname{Ang}(a, b) \in[0, \pi]$ for which $\cos \operatorname{Ang}(a, b)=\langle a, b\rangle /(|a||b|)$, where $\langle a, b\rangle$ denotes the Euclidean inner product of vectors $a$ and $b$.

We cite a lemma for the following analysis (see example 3.16 in [3] (pp. 88)).

Lemma 2.1: $f(z)=|z|_{K}$ is a convex function, where $K$ is a closed convex set in $R^{m}$.

The following properties hold for the projection operator $P_{K}$. Here (i) is the standard non-expansiveness property for convex projection; (ii) comes from exercise 1.2 (c) in [2] (pp. 23) and (iii) is a special case of proposition 1.3 in [2] (pp. 24).

Lemma 2.2: $K$ be a closed convex set in $R^{m}$. Then

(i) $\left|P_{K}(x)-P_{K}(y)\right| \leq|x-y| \forall x, y$;

(ii) $\left.|| x\right|_{K}-|y|_{K}|\leq| x-y \mid \forall x, y$;

(iii) $P_{K}\left(\lambda x+(1-\lambda) P_{K}(x)\right)=P_{K}(x) \forall x, \forall 0<\lambda<1$.

The next lemma can be found in [31].

Lemma 2.3: Let $K$ and $K_{0} \subseteq K$ be two closed convex sets. We have

$$
\left|P_{K}(x)\right|_{K_{0}}^{2}+|x|_{K}^{2} \leq|x|_{K_{0}}^{2} \forall x .
$$

\section{Problem Formulation}

In this section, we introduce the intersection computation problem and the approximately projected algorithm (APCA).

Consider a multi-agent system consisting of $n$ agents with node set $\mathcal{V}=\{1,2, \ldots, n\}$. Each node $i$ is associated with a set $X_{i} \subseteq R^{m}$ and set $X_{i}$ is known only by node $i$. The intersection of all these sets is nonempty, i.e., $\bigcap_{i=1}^{n} X_{i} \neq \emptyset$. Let us denote $X_{0}=\bigcap_{i=1}^{n} X_{i}$. The target of the system is to find a point in $X_{0}$ in a distributed way. For $X_{i}, i=1, \ldots, n$, we use the following assumption:

A1 (Convexity) $X_{i}, i=1, \ldots, n$, are closed convex sets. 


\section{A. Communication Graphs}

The communication over the multi-agent system is modeled as a sequence of directed graphs, $\mathcal{G}_{k}=$ $(\mathcal{V}, \mathcal{E}(k), A(k)), k \geq 0$. We say node $j$ is a neighbor of node $i$ at time $k$ if there is an arc $(i, j) \in \mathcal{E}(k)$, where $a_{i j}(k)$ represents its weight. Let $\mathcal{N}_{i}(k)$ denote the set of neighbors of agent $i$ at time $k$. We introduce an assumption on the weights [26], [31].

A2 (Weights Rule) (i) $\sum_{j \in \mathcal{N}_{i}(k)} a_{i j}(k)=1$ for all $i$ and $k$.

(ii) There exists a constant $0<\eta<1$ such that $a_{i j}(k) \geq \eta$ for all $i, k$ and $j \in \mathcal{N}_{i}(k)$.

For the connectivity of the communication graphs, we introduce the following definition [30], [27].

Definition 3.1: The communication graph is said to be uniformly jointly strongly connected $(U J S C)$ if there exists a positive integer $T$ such that $\mathcal{G}([k, k+T))$ is strongly connected for all $k \geq 0$, where $\mathcal{G}([k, k+T))$ denotes the union graph with node set $\mathcal{V}$ and arc set $\bigcup_{k \leq s<k+T} \mathcal{E}(s)$.

\section{B. Approximate Projection}

Projection methods have been widely used to solve various problems, including projected consensus [27], the convex intersection computation [35], [36] and distributed computation [4]. In the most literature, the projection point $P_{K}(z)$ of $z$ onto closed convex set $K$ is required to achieve desired convergence, but in practice it is hard to be obtained and often is computed approximately. Here is the definition of approximate projection.

Definition 3.2: Suppose $K \subseteq R^{m}$ is a closed convex set and $0<\theta<\pi / 2$. If $v \in K, \mathscr{P}_{K}^{a}(v, \theta)=\{v\}$; if $v \notin K$, we define the approximate projection $\mathscr{P}_{K}^{a}(v, \theta)$ of point $z$ onto $K$ with approximate angle $\theta$ as the following set:

$$
\mathscr{P}_{K}^{a}(v, \theta)=\mathbf{C}_{K}(v, \theta) \bigcap \mathbf{H}_{K}^{+}(v),
$$

where

$$
\begin{aligned}
& \mathbf{C}_{K}(v, \theta)=v+\left\{\left.z\left|\left\langle z, P_{K}(v)-v\right\rangle \geq\right| z|| v\right|_{K} \cos \theta\right\} ; \\
& \mathbf{H}_{K}^{+}(v)=\left\{z \mid\left\langle v-P_{K}(v), z\right\rangle \geq\left\langle v-P_{K}(v), P_{K}(v)\right\rangle\right\} .
\end{aligned}
$$

In fact, $\mathbf{C}_{K}(v, \theta)$ is the convex cone generated by point $v \notin$ $K$ and all vectors having angle with $P_{K}(v)-v$ less than $\theta$ and $\mathbf{H}_{K}^{+}(v)$ is the half-space containing point $v$ with

$$
\mathbf{H}_{K}(v):=\left\{z \mid\left\langle v-P_{K}(v), z\right\rangle=\left\langle v-P_{K}(v), P_{K}(v)\right\rangle\right\}
$$

being a supporting hyperplane to $K$ at $P_{K}(v)$.

\section{Distributed Iterative Algorithm}

To solve the intersection computation problem, we propose the following approximately projected consensus algorithm (APCA):

$$
x_{i}(k+1)=\sum_{j \in \mathcal{N}_{i}(k)} a_{i j}(k) P_{j}^{a}(k)
$$

where $P_{i}^{a}(k) \in \mathscr{P}_{X_{i}}^{a}\left(x_{i}(k), \theta_{k}\right)$ for all $i$ and $k$.

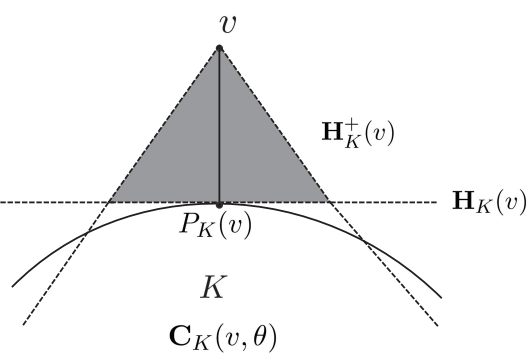

Fig. 1. The set marked by the shaded area is the approximate projection of point $v$ onto closed convex $K$.

Denote $\widehat{P}_{i}^{a}(k)$ as the intersection point of the half-line $\left\{z \mid z=x_{i}(k)+r\left(P_{i}^{a}(k)-x_{i}(k)\right), r \geq 0\right\}$ and the hyperplane $\mathbf{H}_{X_{i}}\left(x_{i}(k)\right)$ if $x_{i}(k) \notin X_{i}$. Therefore, it is easy to see that there exists $0 \leq \alpha_{i, k} \leq 1$ such that

$$
P_{i}^{a}(k)=\left(1-\alpha_{i, k}\right) x_{i}(k)+\alpha_{i, k} \widehat{P}_{i}^{a}(k) .
$$

Combining with (2) and (3), we have

$$
x_{i}(k+1)=\sum_{j \in \mathcal{N}_{i}(k)} a_{i j}(k)\left(\left(1-\alpha_{j, k}\right) x_{j}(k)+\alpha_{j, k} \widehat{P}_{j}^{a}(k)\right),
$$

where if $x_{i}(k) \notin X_{i}, \quad \widehat{P}_{i}^{a}(k) \in \mathbf{H}_{X_{i}}\left(x_{i}(k)\right)$ and $\operatorname{Ang}\left(\widehat{P}_{i}^{a}(k)-x_{i}(k), P_{X_{i}}\left(x_{i}(k)\right)-x_{i}(k)\right) \leq \theta_{k}$.

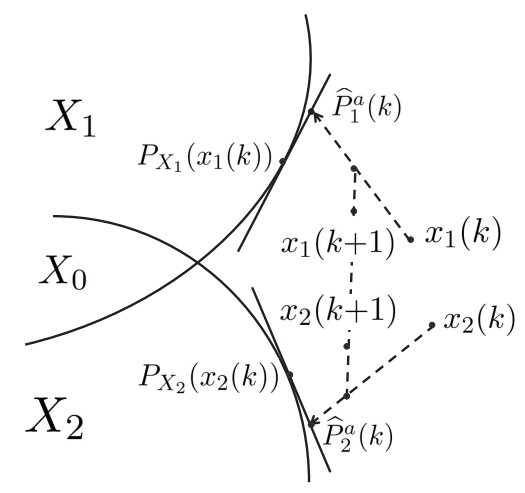

Fig. 2. Approximately projected consensus algorithm

We illustrate the iteration process of APCA in Figure 2. For the approximate angle $\theta_{k}$, we use the following assumption. $\mathbf{A 3} 0 \leq \theta_{k} \leq \theta^{*}<\pi / 2$ for all $k$.

Our problem is introduced in the following definition.

Definition 3.3: A global optimal consensus is achieved for the APCA if, for any initial condition $x(0) \in R^{n m}$, there exists $x^{*} \in X_{0}$ such that

$$
\lim _{k \rightarrow \infty} x_{i}(k)=x^{*}, i=1, \ldots, n .
$$




\section{Main Results and Convergence Analysis}

In this section, we obtain the results on APCA as follows. Denote $\alpha_{k}^{-}=\min _{1 \leq i \leq n} \alpha_{i, k}$ and $\alpha_{k}^{+}=\max _{1 \leq i \leq n} \alpha_{i, k}$.

Theorem 4.1: Suppose A1-A3 hold. Global optimal consensus is achieved for the APCA if

(i) the communication graph is UJSC;

(ii) $\sum_{k=0}^{\infty} \alpha_{k}^{-}=\infty$;

(iii) $\sum_{k=0}^{\infty} \alpha_{k}^{+} \theta_{k}<\infty$.

To investigate the necessity of divergent projection accuracy sum, we impose another assumption on the boundedness of the $n$ sets $X_{i}, i=1, \ldots, n$.

A4 (Compact Sets) $X_{i}, i=1, \ldots, n$, are bounded.

Theorem 4.2: Suppose A1-A4 hold and the communication graph is UJSC. Let $\theta_{k} \equiv 0$. Global optimal consensus is achieved for the APCA if $\sum_{k=0}^{\infty} \alpha_{k}^{-}=\infty$ and only if $\sum_{k=0}^{\infty} \alpha_{k}^{+}=\infty$. Particularly, if there exist $0<\alpha_{k}<0$ such that $\alpha_{i, k}=\alpha_{j, k}=\alpha_{k}$ for all $i, j$ and $k$, then global optimal consensus is achieved for the APCA if and only if $\sum_{k=0}^{\infty} \alpha_{k}=\infty$.

\section{A. Lemmas}

We establish several useful lemmas in this subsection, some proofs are omitted due to space limitations.

Lemma 4.3: For all $i$ and $k \geq s$, we have

$$
\begin{gathered}
\left|x_{i}(k+1)\right|_{X_{0}} \leq \sum_{j \in \mathcal{N}_{i}(k)} a_{i j}(k)\left(\left(1-\alpha_{j, k}\right)\left|x_{j}(k)\right|_{X_{0}}+\alpha_{j, k}\right. \\
\left.\sqrt{\left|x_{j}(k)\right|_{X_{0}}^{2}-\left|x_{j}(k)\right|_{X_{j}}^{2}}+\tan \theta_{k} \alpha_{j, k}\left|x_{j}(k)\right|_{X_{0}}\right)
\end{gathered}
$$

Proof. By Lemma 2.2 (ii), we have

$$
\left|\widehat{P}_{j}^{a}(k)\right|_{X_{0}} \leq\left|\widehat{P}_{j}^{a}(k)-P_{X_{j}}\left(x_{j}(k)\right)\right|+\left|P_{X_{j}}\left(x_{j}(k)\right)\right|_{X_{0}} .
$$

The definition of $\widehat{P}_{j}^{a}(k)$ ensures that

$$
\left|\widehat{P}_{j}^{a}(k)-P_{X_{j}}\left(x_{j}(k)\right)\right| \leq \tan \theta_{k}\left|x_{j}(k)\right|_{X_{j}} .
$$

Moreover, it follows from Lemma 2.3 that for any $j \in \mathcal{V}$,

$$
\left|P_{X_{j}}\left(x_{j}(k)\right)\right|_{X_{0}} \leq \sqrt{\left|x_{j}(k)\right|_{X_{0}}^{2}-\left|x_{j}(k)\right|_{X_{j}}^{2}} .
$$

By applying Lemma 2.1 for (4) and noting inequalities (6), (7) and (8), the conclusion follows.

Lemma 4.4: For any $z \in X_{0}$, we have for all $k$,

$$
\max _{1 \leq i \leq n}\left|x_{i}(k+1)-z\right| \leq e^{\sum_{l=0}^{\infty} \alpha_{l}^{+} \tan \theta_{l}} \max _{1 \leq i \leq n}\left|x_{i}(0)-z\right| .
$$

$1 \leq i \leq n$
The next lemma is a special case of various random versions, for example, see Lemma 11 in [6] (pp. 50).

Lemma 4.5: Let $\left\{a_{k}\right\}_{k=0}^{\infty}$ and $\left\{b_{k}\right\}_{k=0}^{\infty}$ be non-negative sequences with $\sum_{k=0}^{\infty} b_{k}<\infty$. Suppose

$$
a_{k+1} \leq a_{k}+b_{k} \text { for all } k
$$

Then $\lim _{k \rightarrow \infty} a_{k}$ is a finite number.

It is easy to see that $\tan \theta \leq\left(\tan \theta^{*} / \theta^{*}\right) \theta$ for $0 \leq \theta \leq \theta^{*}$. Thus, if $\sum_{k=0}^{\infty} \alpha_{k}^{+} \theta_{k}<\infty, \sum_{k=0}^{\infty} \alpha_{k}^{+} \tan \theta_{k}<\infty$ and then $\left\{x_{i}(k), i \in \mathcal{V}\right\}_{k=0}^{\infty}$ is bounded by Lemma 4.4. By Lemmas 4.3, 4.4 and 4.5, we have the following lemma.

Lemma 4.6: If $\sum_{k=0}^{\infty} \alpha_{k}^{+} \theta_{k}<\infty$, the following limit exists

\section{Denote}

$$
\vartheta:=\lim _{k \rightarrow \infty} \max _{1 \leq i \leq n}\left|x_{i}(k)\right|_{X_{0}} .
$$

$$
\eta_{i}^{+}=\limsup _{k \rightarrow \infty}\left|x_{i}(k)\right|_{X_{0}}, \quad \eta_{i}^{-}=\liminf _{k \rightarrow \infty}\left|x_{i}(k)\right|_{X_{0}}, i \in \mathcal{V} .
$$

Obviously, $0 \leq \eta_{i}^{-} \leq \eta_{i}^{+} \leq \vartheta$ for all $i$.

Lemma 4.7: Suppose the communication graph is UJSC, A2 holds, $\sum_{k=0}^{\infty} \alpha_{k}^{+} \theta_{k}<\infty$ and there exists some agent $i_{0} \in \mathcal{V}$ such that $\eta_{i_{0}}^{-}<\vartheta$. Then $\vartheta=0$.

The next lemma can be obtained by combining Lemma 2 in [26].

Lemma 4.8: If the communication graph is UJSC and A2 holds, then every entry of $\Phi(k, s)$ is not less than $\eta^{\hat{T}}$ for all $s$ and $k \geq s+\hat{T}-1$, where $\hat{T}=(n-1) T, T$ is the constant in Definition 3.1 and $\eta$ is the lower bound of weights in $\mathbf{A 2}$.

Lemma 4.9:

$$
\frac{1}{n} \sum_{i=1}^{n} \sqrt{\bar{v}^{2}-v_{i}^{2}} \leq \sqrt{\bar{v}^{2}-\left(\frac{\sum_{i=1}^{n} v_{i}}{n}\right)^{2}},
$$

where $\bar{v} \geq v_{i} \geq 0$ for all $i$.

Consider the following consensus model with noise $w_{i}$,

$$
z_{i}(k+1)=\sum_{j \in \mathcal{N}_{i}(k)} b_{i j}(k) z_{j}(k)+w_{i}(k), i=1, \ldots, n,
$$

where $\left\{b_{i j}(k), i, j \in \mathcal{V}, k \geq 0\right\}$ satisfy A2. The next lemma can be obtained from Theorem 1 in [33].

Lemma 4.10: If the communication graph of system (9) is UJSC with $\lim _{k \rightarrow \infty} w_{i}(k)=0$ for all $i$, then consensus is achieved for system (9).

\section{B. Proofs}

In this subsection, we present the proofs of Theorems 4.1 and 4.2.

1) Proof of Theorem 4.1: Rewrite (4) as

$$
\begin{aligned}
& x_{i}(k+1)=\sum_{j \in \mathcal{N}_{i}(k)} a_{i j}(k) x_{j}(k)+\sum_{j \in \mathcal{N}_{i}(k)} a_{i j}(k) \alpha_{j, k} \\
& \left(\left(P_{X_{j}}\left(x_{j}(k)\right)-x_{j}(k)\right)+\left(\widehat{P}_{j}^{a}(k)-P_{X_{j}}\left(x_{j}(k)\right)\right)\right) .
\end{aligned}
$$

Based on (7), the second term in last equality is not greater than

$$
\max _{1 \leq i \leq n} \alpha_{i, k}\left|x_{i}(k)\right|_{X_{i}}+\alpha_{k}^{+} \tan \theta_{k} \max _{1 \leq i \leq n}\left|x_{i}(k)\right|_{X_{i}} .
$$

Note that $\vartheta=0$ leads to $\lim _{k \rightarrow \infty} \max _{1 \leq i \leq n}\left|x_{i}(k)\right|_{X_{i}} \leq$ $\lim _{k \rightarrow \infty} \max _{1 \leq i \leq n}\left|x_{i}(k)\right|_{X_{0}}=0$ and then the term in (11) tends to zero as $k \rightarrow \infty$. Therefore, by applying Lemma 4.10 for (10), we have that if $\vartheta=0$, then the consensus is achieved. 
Moreover, we claim that if $\vartheta=0$ and the consensus is achieved, then all agents will converge to a point in $X_{0}$. Since $\left\{x_{i}(k), i \in \mathcal{V}\right\}_{k=0}^{\infty}$ is bounded by Lemma 4.4 and the consensus is achieved, there is $x^{*} \in X_{0}$ and a subsequence $\left\{k_{l}\right\}_{l=1}^{\infty}$ such that $\lim _{l \rightarrow \infty} x_{i}\left(k_{l}\right)=x^{*}$ for all $i$. Similar with Lemma 4.4, we have

$$
\max _{1 \leq i \leq n}\left|x_{i}(k)-x^{*}\right| \leq e^{\sum_{p=0}^{\infty} \alpha_{p}^{+} \tan \theta_{p}} \max _{1 \leq i \leq n}\left|x_{i}\left(k_{l}\right)-x^{*}\right|
$$

for $k \geq k_{l}$, which implies $\lim _{k \rightarrow \infty} x_{i}(k)=x^{*}$ for all $i$.

If there exists some agent $i_{0}$ such that $\eta_{i_{0}}^{-}<\vartheta$, then by Lemma $4.7, \vartheta=0$. Therefore, we only need to prove $\vartheta=0$ when $\eta_{i}^{+}=\eta_{i}^{-}=\vartheta$ for all $i$, which shall be proven by contradiction. If $\vartheta>0$, then for any $\varepsilon>0$, there exist $K_{0}=K_{0}(\varepsilon)$ such that $\left|x_{i}(k)\right|_{X_{0}} \leq \vartheta+\varepsilon$ and $d_{0} \alpha_{k}^{+} \theta_{k} \leq \varepsilon$ for $k \geq K_{0}$ and all $i$, where $d_{0}=$ $\left(\tan \theta^{*} / \theta^{*}\right) \sup _{1 \leq i \leq n, k \geq 0}\left|x_{i}(k)\right|_{X_{0}}$. We complete the proof by the following two steps.

(i) Suppose $\eta_{i}^{+}=\eta_{i}^{-}=\vartheta$ for all $i$. The consensus is achieved: $\lim _{k \rightarrow \infty}\left|x_{i}(k)-x_{j}(k)\right|=0$ for all $i, j$.

Denote

$$
\varsigma_{i}=\limsup _{k \rightarrow \infty} \alpha_{i, k}\left|x_{i}(k)\right|_{X_{i}}, i \in \mathcal{V} .
$$

We prove $\varsigma_{i}=0$ for all $i$ by contradiction. If there exists some agent $i_{0}$ such that $\varsigma_{i_{0}}>0$, then there is an increasing time subsequence $\left\{k_{l}\right\}_{l=1}^{\infty}$ with $k_{1} \geq K_{0}$ such that $\alpha_{i_{0}, k_{l}}\left|x_{i_{0}}\left(k_{l}\right)\right|_{X_{i_{0}}} \geq c \varsigma_{i_{0}}$ for all $l$ and some $0<c<1$. Therefore, by (5) we have

$$
\begin{aligned}
\left|x_{i_{0}}\left(k_{l}+1\right)\right|_{X_{0}} \leq & \left(1-\eta \alpha_{i_{0}, k_{l}}\right)(\vartheta+\varepsilon) \\
& +\eta \sqrt{\alpha_{i_{0}, k_{l}}^{2}(\vartheta+\varepsilon)^{2}-c^{2} \varsigma_{i_{0}}^{2}}+\varepsilon
\end{aligned}
$$

which yields a contradiction since the right hand side of (12) is less than $\vartheta$ for sufficiently small $\varepsilon$ and sufficiently large $l$.

Thus, $\lim _{k \rightarrow \infty} \alpha_{i, k}\left|x_{i}(k)\right|_{X_{i}}=0$ for all $i$. Moreover, since $\sum_{k=0}^{\infty} \alpha_{k}^{+} \theta_{k}<\infty, \lim _{k \rightarrow \infty} \alpha_{k}^{+} \tan \theta_{k} \leq$ $\left(\tan \theta^{*} / \theta^{*}\right) \lim _{k \rightarrow \infty} \alpha_{k}^{+} \theta_{k}=0$. The two preceding conclusions and the boundedness of $\left\{x_{i}(k), i \in \mathcal{V}\right\}_{k=0}^{\infty}$ imply that the term in (11) tends to zero and then the consensus is achieved by applying Lemma 4.10 for (10) again.

(ii) Suppose $\eta_{i}^{+}=\eta_{i}^{-}=\vartheta$ for all $i$. All agents converge to the optimal set: $\lim _{k \rightarrow \infty}\left|x_{i}(k)\right|_{X_{0}}=0$ for all $i$.

Denote

$$
\delta=\liminf _{k \rightarrow \infty} \sum_{i=1}^{n}\left|x_{i}(k)\right|_{X_{i}} .
$$

We prove that $\delta=0$ by contradiction. Otherwise, suppose $\delta>0$.

Denote $D_{k}=\operatorname{diag}\left\{\alpha_{1, k}, \alpha_{2, k}, \ldots, \alpha_{n, k}\right\},|x(k)|_{X_{0}}=$ $\left(\left|x_{1}(k)\right|_{X_{0}}, \ldots,\left|x_{n}(k)\right|_{X_{0}}\right)^{T}$ and $y(k)=\left(y_{1}(k), \ldots, y_{n}(k)\right)^{T}$,

$$
y_{i}(k)=\left|x_{i}(k)\right|_{X_{0}}-\sqrt{\left|x_{i}(k)\right|_{X_{0}}^{2}-\left|x_{i}(k)\right|_{X_{i}}^{2}}, i \in \mathcal{V} .
$$

From inequality (5), we have for $k \geq s$,

$$
\begin{aligned}
|x(k+1)|_{X_{0}} & \leq \Phi(k, s)|x(s)|_{X_{0}} \\
& -\sum_{l=s}^{k-\hat{T}-1} \Phi(k, l) D_{l} y(l)+d_{0} \sum_{l=s}^{k} \alpha_{l}^{+} \theta_{l}
\end{aligned}
$$

where $\hat{T}=(n-1) T$ and $\Phi(k, s)=A(k) \cdots A(s+1) A(s)$.

For $\bar{\varepsilon}=\delta^{2} /\left(4 n^{2} \vartheta+2 \delta\right)$, there exists sufficiently large $K_{1}$ such that $\sum_{i=1}^{n}\left|x_{i}(k)\right|_{X_{i}}>\delta-\bar{\varepsilon}$ and $\vartheta-\bar{\varepsilon} \leq\left|x_{i}(k)\right|_{X_{0}} \leq$ $\vartheta+\bar{\varepsilon}$ for $k \geq K_{1}$. For $k \geq K_{1}$, from Lemma 4.9 we have

$$
\begin{aligned}
& \sum_{i=1}^{n}\left(\left|x_{i}(k)\right|_{X_{0}}-\sqrt{\left|x_{i}(k)\right|_{X_{0}}^{2}-\left|x_{i}(k)\right|_{X_{i}}^{2}}\right) \\
& \geq n\left(\vartheta-\bar{\varepsilon}-\sqrt{(\vartheta+\bar{\varepsilon})^{2}-((\delta-\bar{\varepsilon}) / n)^{2}}\right):=\zeta>0 .
\end{aligned}
$$

Namely, $\sum_{i=1}^{n} y_{i}(l) \geq \zeta$ for $l \geq K_{1}$. Combining the preceding inequality with Lemma 4.8 yields that every component of $\Phi(k, l) D_{l} y(l)$ is not less than $\eta^{\hat{T}} \zeta \alpha_{l}^{-}$for $K_{1} \leq l \leq$ $k-\hat{T}-1$. Then by (13) with taking $s=K_{1}$, we obtain

$$
\begin{aligned}
|x(k+1)|_{X_{0}} & \leq \Phi\left(k, K_{1}\right)\left|x\left(K_{1}\right)\right|_{X_{0}} \\
& -\eta^{\hat{T}} \zeta \sum_{l=K_{1}}^{k-\hat{T}-1} \alpha_{l}^{-} \mathbf{1}+d_{0} \sum_{l=K_{1}}^{k} \alpha_{l}^{+} \theta_{l}
\end{aligned}
$$

where $\mathbf{1}$ is the vector of all ones. Note that $\sum_{l=K_{1}}^{\infty} \alpha_{l}^{-}=$ $\infty, \sum_{l=K_{1}}^{\infty} \alpha_{l}^{+} \theta_{l}<\infty$ and $\lim _{k \rightarrow \infty}|x(k)|_{X_{0}}=\vartheta \mathbf{1}$, a contradiction will yield by taking the limit as $k \rightarrow \infty$ in (14).

Therefore, $\delta=\liminf _{k \rightarrow \infty} \sum_{i=1}^{n}\left|x_{i}(k)\right|_{X_{i}}=0$, that is, there is a subsequence $\left\{k_{l}\right\}_{l=0}^{\infty}$ such that $\lim _{l \rightarrow \infty} \sum_{i=1}^{n}\left|x_{i}\left(k_{l}\right)\right|_{X_{i}}=0$. Since the consensus is achieved by what we have proven in the first step (i), we have

$$
\lim _{l \rightarrow \infty} \sum_{i=1}^{n}\left|x_{i}\left(k_{l}\right)\right|_{X_{j}}=0 \text { for all } j \in \mathcal{V}
$$

which implies $\vartheta=\lim _{l \rightarrow \infty} \max _{1 \leq i \leq n}\left|x_{i}\left(k_{l}\right)\right|_{X_{0}}=0$.

2) Proof of Theorem 4.2: The sufficiency has been obtained in Theorem 4.1, here we focus on the necessity. It is easy to find that if $\theta_{k} \equiv 0$, the intersection set in (1) is the line segment from $x_{i}(k)$ to $P_{X_{i}}\left(x_{i}(k)\right)$ and then $\widehat{P}_{i}^{a}(k)=P_{X_{i}}\left(x_{i}(k)\right)$.

Denote $d^{*}:=\sup _{y_{1}, y_{2} \in \bigcup_{i=1}^{n} X_{i}}\left|y_{1}-y_{2}\right|$, which is finite since $X_{i}, i=1, \ldots, n$ are bounded. We next prove that if $\sum_{k=0}^{\infty} \alpha_{k}^{+}<\infty$, then there exist initial conditions from which all agents will not converge to set $X_{0}$. Let $\bar{x} \in R^{m}$, which will be selected later, and $x_{i}(0)=\bar{x}$ for all $i \in \mathcal{V}$. 
By (4), $x_{i}(1)$ can be rewritten as

$$
\begin{aligned}
x_{i}(1) & =\sum_{j \in \mathcal{N}_{i}(0)} a_{i j}(0)\left(\left(1-\alpha_{j, 0}\right) x_{j}(0)+\alpha_{j, 0} P_{X_{j}}\left(x_{j}(0)\right)\right) \\
& =\sum_{j \in \mathcal{N}_{i}(0)} a_{i j}(0)\left(\left(1-\alpha_{j, 0}\right) \bar{x}+\alpha_{j, 0} P_{X_{0}}(\bar{x})\right)+\Delta_{i 0}, \\
& =\left(1-\beta_{i, 0}\right) \bar{x}+\beta_{i, 0} P_{X_{0}}(\bar{x})+\Delta_{i 0},
\end{aligned}
$$

where $1-\beta_{i, 0}=\sum_{j \in \mathcal{N}_{i}(0)} a_{i j}(0)\left(1-\alpha_{j, 0}\right)$ and $\Delta_{i 0}=$ $\sum_{j \in \mathcal{N}_{i}(0)} a_{i j}(0) \alpha_{j, 0}\left(P_{X_{j}}(\bar{x})-P_{X_{0}}(\bar{x})\right)$ with $\left|\Delta_{i 0}\right| \leq \alpha_{0}^{+} d^{*}$ for all $i$.

We also have

$$
\begin{aligned}
& x_{i}(2)=\sum_{j \in \mathcal{N}_{i}(1)} a_{i j}(1)\left(\left(1-\alpha_{j, 1}\right) x_{j}(1)+\alpha_{j, 1} P_{X_{j}}\left(x_{j}(1)\right)\right) \\
& =\sum_{j \in \mathcal{N}_{i}(1)} a_{i j}(1)\left(1-\alpha_{j, 1}\right)\left(\left(1-\beta_{j, 0}\right) \bar{x}+\beta_{j, 0} P_{X_{0}}(\bar{x})\right) \\
& +\Delta_{i 1}+\sum_{j \in \mathcal{N}_{i}(1)} a_{i j}(1) \alpha_{j, 1} P_{X_{0}}\left(\left(1-\beta_{j, 0}\right) \bar{x}+\beta_{j, 0} P_{X_{0}}(\bar{x})\right) \\
& =\left(1-\beta_{i, 1}\right) \bar{x}+\beta_{i, 1} P_{X_{0}}(\bar{x})+\Delta_{i 1},
\end{aligned}
$$

where $1-\beta_{i, 1}=\sum_{j \in \mathcal{N}_{i}(1)} a_{i j}(1)\left(1-\alpha_{j, 1}\right)\left(1-\beta_{j, 0}\right)$, the third equality follows from Lemma 2.2 (iii) and $\Delta_{i 1}=\Delta_{i 1}^{1}+$ $\Delta_{i 1}^{2}+\Delta_{i 1}^{3}$ with $\Delta_{i 1}^{1}=\sum_{j \in \mathcal{N}_{i}(1)} a_{i j}(1)\left(1-\alpha_{j, 1}\right) \Delta_{j 0}$;

$$
\begin{aligned}
\Delta_{i 1}^{2}= & \sum_{j \in \mathcal{N}_{i}(1)} a_{i j}(1) \alpha_{j, 1}\left(P_{X_{j}}\left(x_{j}(1)\right)-P_{X_{0}}\left(x_{j}(1)\right)\right) \\
\Delta_{i 1}^{3}= & \sum_{j \in \mathcal{N}_{i}(1)} a_{i j}(1) \alpha_{j, 1}\left(P_{X_{0}}\left(x_{j}(1)\right)\right. \\
& \left.\quad-P_{X_{0}}\left(\left(1-\beta_{j, 0}\right) \bar{x}+\beta_{j, 0} P_{X_{0}}(\bar{x})\right)\right) .
\end{aligned}
$$

Lemma 2.2 (i) implies that $\left|\Delta_{i 1}^{1}+\Delta_{i 1}^{3}\right| \leq \max _{1 \leq i \leq n}\left|\Delta_{i 0}\right| \leq$ $\alpha_{0}^{+} d^{*}$ and then $\left|\Delta_{i 1}\right| \leq\left|\Delta_{i 1}^{1}+\Delta_{i 1}^{3}\right|+\left|\Delta_{i 1}^{2}\right| \leq\left(\alpha_{0}^{+}+\alpha_{1}^{+}\right) d^{*}$ for all $i$.

Similarly, we can show by induction that for all $i$ and $k$, $x_{i}(k+1)$ can be expressed as

$$
x_{i}(k+1)=\left(1-\beta_{i, k}\right) \bar{x}+\beta_{i, k} P_{X_{0}}(\bar{x})+\Delta_{i k},
$$

where $\left|\Delta_{i k}\right| \leq \sum_{l=0}^{k} \alpha_{l}^{+} d^{*}$ and $\left\{\beta_{i, k}, i \in \mathcal{V}\right\}_{k=0}^{\infty}$ satisfy

$$
1-\beta_{i, k}=\sum_{j \in \mathcal{N}_{i}(k)} a_{i j}(k)\left(1-\alpha_{j, k}\right)\left(1-\beta_{j, k-1}\right) .
$$

Based on (16), we can show by induction that

$$
1-\beta_{i, k} \geq \prod_{l=0}^{k}\left(1-\alpha_{l}^{+}\right) \text {for all } i \text { and } k .
$$

It follows from (15), Lemma 2.2 (ii), (iii) and (17) that

$$
\left|x_{i}(k+1)\right|_{X_{0}} \geq \prod_{l=0}^{k}\left(1-\alpha_{l}^{+}\right)|\bar{x}|_{X_{0}}-\left|\Delta_{i k}\right| .
$$

Taking the inferior limit on the two sides in (18), we have

$$
\liminf _{k \rightarrow \infty}\left|x_{i}(k)\right|_{X_{0}} \geq \prod_{l=0}^{\infty}\left(1-\alpha_{l}^{+}\right)|\bar{x}|_{X_{0}}-\sum_{l=0}^{\infty} \alpha_{l}^{+} d^{*},
$$

which is positive provided that

$$
|\bar{x}|_{X_{0}}>\frac{\sum_{l=0}^{\infty} \alpha_{l}^{+} d^{*}}{\prod_{l=0}^{\infty}\left(1-\alpha_{l}^{+}\right)}
$$

where $\prod_{l=0}^{\infty}\left(1-\alpha_{l}^{+}\right)>0$ since $\sum_{l=0}^{\infty} \alpha_{l}^{+}<\infty$. Thus, all agents can not achieve an optimal consensus for all initial conditions satisfying (19). We complete the proof.

\section{A NUMERICAL EXAMPLE}

Example 5.1: The multi-agent system consists of three agents 1,2 and 3 in $R^{2}$ with fixed graph, where $X_{1}, X_{2}$ and $X_{3}$ are three balls with centers $(1,0),(-1,0),(0,-1)$ and radius $1 ; X_{0}=\{(0,0)\} ; \theta_{k} \equiv 0$; weights $a_{11}=$ $a_{12}=a_{22}=a_{23}=a_{31}=a_{33}=0.5$; the initial condition $x_{1}(0)=(-0.5,-0.5), x_{2}(0)=(0.5,-0.5)$ and $x_{3}(0)=$ $(0.5,1.5)$. Here $h(k)=\max _{1 \leq i \leq 3}\left|x_{i}(k)\right|_{X_{0}}$. The following figure shows that the APCA $\left(\alpha_{i, k}=0.5\right.$ for all $i$ and $\left.k\right)$ converges faster than the exact projected consensus algorithm $\left(\alpha_{i, k}=1\right.$ for all $i$ and $k$ ).

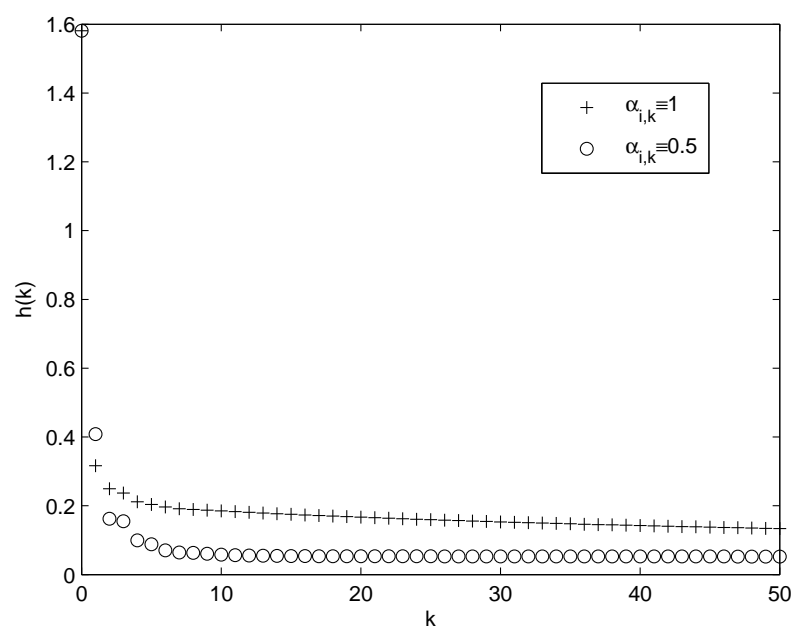

\section{Conclusions}

In this paper, we presented an approximately projected consensus algorithm (APCA) for a multi-agent system to cooperatively compute the intersection of a serial of convex sets, each of which is known only to a particular node. We allowed each node to only compute an approximate projection. Sufficient and/or necessary conditions were obtained for the considered algorithm on how much projection accuracy is required to ensure a global consensus within the intersection set, under the assumption that the communication graph is uniformly jointly strongly connected. A numerical example 
was also given indicating that the APCA sometimes achieves better performance than the exact projected consensus algorithm. This implied that, individual optimum seeking may not be so important for optimizing the collective objective.

\section{REFERENCES}

[1] R. T. Rockafellar, Convex Analysis. New Jersey: Princeton University Press, 1972.

[2] F. Clarke, Yu.S. Ledyaev, R. Stern and P. Wolenski, Nonsmooth Analysis and Control Theory. Speringer-Verlag, 1998.

[3] S. Boyd and L. Vandenberghe, Convex Optimization. New York: Cambridge University Press, 2004.

[4] D. Bertsekas and J. Tsitsiklis, Parallel and Distributed Computation: Numerical Methods. Princeton Hall, 1989.

[5] C. Godsil and G. Royle, Algebraic Graph Theory. Springer-Verlag, New York, 2001.

[6] B. T. Polyak, Introduction to Optimization. Optimization Software, Inc., New York, 1987.

[7] J. Tsitsiklis, D. Bertsekas, and M. Athans, "Distributed asynchronous deterministic and stochastic gradient optimization algorithms," IEEE Trans. Autom. Control, vol. 31, no. 9, pp. 803-812, 1986.

[8] A. Jadbabaie, J. Lin, and A. S. Morse, "Coordination of groups of mobile agents using nearest neighbor rules," IEEE Trans. Autom. Control, vol. 48, no. 6, pp. 988-1001, 2003.

[9] S. Muthukrishnan, B. Ghosh, and M. Schultz, "First and second order diffusive methods for rapid, coarse, distributed load balancing," Theory of Computing Systems, vol. 31, pp. 331-354, 1998.

[10] R. Diekmann, A. Frommer, and B. Monien, "Efficient schemes for nearest neighbor load balancing," Parallel Computing, vol. 25, pp. 789812, 1999.

[11] G. Shi and Y. Hong, "Global target aggregation and state agreement of nonlinear multi-agent systems with switching topologies," Automatica, vol. 45 , no. $5,1165-1175,2009$.

[12] G. Shi, Y. Hong and K. H. Johansson, "Connectivity and set tracking of multi-agent systems guided by multiple moving leaders," IEEE Trans. Autom. Control, vol. 57, no. 3, pp. 663-676, 2012.

[13] M. H. DeGroot, "Reaching a consensus," Journal of the American Statistical Association, vol. 69, no. 345, pp. 118-121, 1974.

[14] D. Acemoglu, A. Ozdaglar and A. ParandehGheibi, "Spread of (mis)information in social networks," Games and Economic Behavior, vol. 70, no. 2, 194-227, 2010.

[15] W. Ren and R. Beard, "Consensus seeking in multi-agent systems under dynamically changing interaction topologies," IEEE Trans. Autom. Control, vol. 50, no. 5, 655-661.

[16] S. Martinez, J. Cortes, and F. Bullo, "Motion coordination with distributed information," IEEE Control Syst. Mag., vol. 27, no. 4, pp. 75-88, 2007.

[17] R. Olfati-Saber, "Flocking for multi-agent dynamic systems: algorithms and theory," IEEE Trans. Autom. Control, vol. 51, no. 3, pp. 401-420, 2006.

[18] M. Cao, A. S. Morse and B. D. O. Anderson, "Reaching a consensus in a dynamically changing environment: a graphical approach," SIAM J. Control Optim., vol. 47, no. 2, 575-600, 2008.
[19] H. G. Tanner, A. Jadbabaie, G. J. Pappas, "Flocking in fixed and switching networks," IEEE Trans. Autom. Control, vol. 52, no.5, pp. 863-868, 2007.

[20] M. Rabbat and R. Nowak, "Distributed optimization in sensor networks," in IPSN'04, pp. 20-27, 2004.

[21] S. S. Ram, A. Nedić, and V. V. Veeravalli, "Stochastic incremental gradient descent for estimation in sensor networks," in Proc. Asilomar Conference on Signals, Systems, and Computers, Pacific Grove, pp. 582586, 2007.

[22] B. Johansson, T. Keviczky, M. Johansson, and K. H. Johansson, "Subgradient methods and consensus algorithms for solving convex optimization problems," in Proc. IEEE Conference on Decision and Control, Cancun, Mexico, pp. 4185-4190, 2008.

[23] A. Nedić, A. Olshevsky, A. Ozdaglar, and J. N. Tsitsiklis, "Distributed subgradient methods and quantization effects," in Proc. IEEE Conf. on Decision and Control, Cancun, Mexico, pp. 4177-4184, 2008.

[24] S. S. Ram, A. Nedić, and V. V. Veeravalli, "Incremental stochastic subgradient algorithms for convex optimization," SIAM Journal on Optimization, vol. 20, no. 2, 691-717, 2009.

[25] B. Johansson, M. Rabi and M. Johansson, "A randomized incremental subgradient method for distributed optimization in networked systems," SIAM Journal on Optimization, vol. 20, no. 3, pp. 1157-1170, 2009.

[26] A. Nedić and A. Ozdaglar, "Distributed subgradient methods for multiagent optimization," IEEE Trans. Autom. Control, vol. 54, no. 1, pp. 48-61, 2009.

[27] A. Nedić, A. Ozdaglar and P. A. Parrilo, "Constrained consensus and optimization in multi-agent networks," IEEE Trans. Autom. Control, vol. 55, no. 4, pp. 922-938, 2010.

[28] J. Lu, C. Y. Tang, P. R. Regier, and T. D. Bow, "Gossip algorithms for convex consensus optimization over networks," IEEE Trans. Autom. Control, vol. 56, no. 12, pp. 2917-2923, 2011.

[29] J. Lu, P. R. Regier, and C. Y. Tang, "Control of distributed convex optimization," Proc. IEEE Conference on Decision and Control, Atlanta, pp. 489-495, 2010.

[30] G. Shi, K. H. Johansson, and Y. Hong, "Multi-agent systems reaching optimal consensus with directed communication graphs," Proc. American Control Conference, San Francisco, pp. 5456-5461, 2011.

[31] G. Shi and K. H. Johansson, "Randomized optimal consensus of multiagent systems," http://arxiv.org/abs/1108.3223.

[32] D. Jakovetic, J. Xavier, and J. M. F Moura, "Cooperative convex optimization in networked systems: augmented lagrangian algorithms with directed gossip communication," http://arxiv.org/abs/1007.3706.

[33] L. Wang and L. Guo, "Robust consensus and soft control of multi-agent systems with noises," Journal of Systems Science and Complexity, vol. 21, no.3, pp. 406-415, 2008.

[34] N. Aronszajn, "Theory of reproducing kernels," Trans. Amer. Math. Soc., vol. 68 , no. 3 , pp. 337-404, 1950.

[35] L. Gubin, B. Polyak, and E. Raik, "The method of projections for finding the common point of convex sets," U.S.S.R Comput. Math. Math. Phys., vol. 7, no. 6, pp. 1211-1228, 1967.

[36] F. Deutsch, "Rate of convergence of the method of alternating projections," in Parametric Optimization and Approximation, B. Brosowski and F. Deutsch, Eds. Basel, Switzerland: Birkhäuser, vol. 76, pp. 96-107, 1983. 